\title{
Multiple oscillation of whispering-gallery-mode dye lasers in green and orange regions using mixed-dye-doped solid microspheres
}

\author{
Hiroshi Taniguchi \\ Department of Electrical Engineering, Faculty of Engineering, Iwate University, 4-3-5 Ueda, \\ Morioka-shi 020, Japan \\ Shinji Tanosaki \\ Department of Electronic Engineering, Faculty of Engineering, Iwate University, 4-3-5 Ueda, \\ Morioka-shi 020, Japan \\ Hiroshi Yamada and Tamiya Fujiwara \\ Department of Electrical Engineering, Faculty of Engineering, Iwate University, 4-3-5 Ueda, \\ Morioka-shi 020, Japan \\ Mamoru Baba \\ Department of Electronic Engineering, Faculty of Engineering, Iwate University, 4-3-5 Ueda, \\ Morioka-shi 020, Japan
}

(Received 24 September 1992; accepted for publication 15 February 1993)

\begin{abstract}
Mixed-dye-doped solid microspheres are excited by a transversely excited-atmospheric ultraviolet $\mathrm{N}_{2}$ laser to obtain a multiple oscillation of a whispering-gallery-mode (WGM) dye laser in both green and orange regions. The multiple oscillation exhibits separate modes of WGMs in both spectral regions.
\end{abstract}

Several investigators have reported the occurrence of spectrally narrow resonances in the light emitted from transparent or weakly absorbing microspheres when the wavelength of the incident or wavelength-shifted radiation is such that the electromagnetic modes of the microsphere are excited. These resonances have been referred to as whispering-gallery-modes (WGMs), ${ }^{1}$ or morphologydependent resonances (MDRs).

Recently, the advantages of the spherical resonator have been pointed out because of the creation of the highquality factor ( $Q$ value) of electromagnetic modes in the dielectric sphere with micrometer-sized droplets ${ }^{2,3}$ or solid particles. ${ }^{4,5}$ In addition, the high $Q$ value of spherical cavities has been shown to provide us very low-threshold lasers. ${ }^{2,3}$ In this communication, we report briefly the observation of multiple oscillation of WGM dye lasers using mixed-dye-doped solid microspheres pumped by a transversely excited-atmospheric (TEA) ultraviolet (UV) $\mathrm{N}_{2}$ laser, carried out for obtaining fundamental characteristics between dyes doped in microspheres, such as energy transfer effects. The multiple oscillation in both green and orange spectral regions of a WGM dye laser using solid microspheres is one of the recent developments.

A schematic diagram of an experimental setup is shown in Fig. 1. A TEA UV $\mathrm{N}_{2}$ laser ( $337 \mathrm{~nm}$ ) is used to excite a spherical dye-doped WGM laser (DD-WGM), focusing the pumping UV $\mathrm{N}_{2}$ laser beam with a quartz spherical lens (QSL) after beamshaping through a circular beam aperture (CA). The DD-WGM is placed at the position of the larger diameter of the focused beam than the diameter of the sphere.

Emission light from the DD-WGM laser is detected by a detector system (spectrum analyzer or biplanar phototube/oscilloscope system) via quartz-glass optical fi- ber. The position of the DD-WGM laser, which is on a glass plate under a microscope, is adjusted finely in the vicinity of the focal point of the QSL with $5 \mathrm{~cm}$ focal length.

The DD-WGM lasers are prepared by dying the polystyrene or ion-exchange resin [with small diameter $(\sim 20$ $800 \mu \mathrm{m}$ )] in the mixed solutions of rhodamine 101 (RH101) and fluorescein (sodium salt) (NA-F) ethanol solutions $\left(10^{-5}-10^{-3} \mathrm{M}\right)$, for longer and shorter spectral regions, respectively.

The laser-pulse wave forms of the TEA UV $\mathrm{N}_{2}$ laser and DD-WGM laser were measured with a calibrated Hamamatsu Photonics R1193U-02 biplanar phototube and a Hewlett Packard 1744A storage oscilloscope (100 MHz) system. The spectral characteristics of the DD-WGM laser output pumped by the TEA UV $\mathrm{N}_{2}$ laser were analyzed with an optical multichannel analyzer (OMA; Hamamatsu Photonics PMA-10).

The TEA UV $\mathrm{N}_{2}$ laser typically exhibits $1.3 \mathrm{~ns}$ FWHM and $3 \mathrm{~kW}$ peak power at $337 \mathrm{~nm}$ in the second positive band of $\mathrm{N}_{2}$ molecules (measured with a $275 \mathrm{MHz}$ real-time oscilloscope and with a Photodyne 66XLA power/energy meter, respectively).

By finely adjusting the position of the dye-doped sphere in the vicinity of the focal point of the QSL, it was observed that the rim of the dye-doped sphere above threshold is brighter than the center, while the image of the emission pattern is rather uniform below threshold. These spatial images of the emission suggest the features of WGM lasing with high $Q$ values. ${ }^{5}$

In the measurements with a biplanar phototube and a $100 \mathrm{MHz}$ storage oscilloscope system, the pulses between laser and fluorescence (corresponding to the higher and lower $\mathrm{N}_{2}$ laser pumping intensities by neutral density fil- 


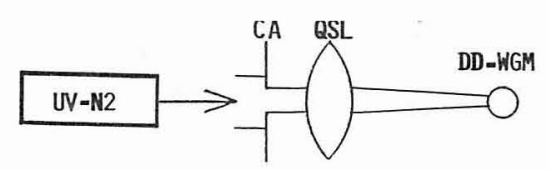

FIG. 1. Experimental setup of WGM dye laser system. UV $\mathrm{N}_{2}$ : TEA UV $\mathrm{N}_{2}$ laser; CA: circular beam-aperture; QSL: quartz spherical lens; DDWGM: spherical mixed-dye-doped WGM laser.

ters) were clearly different with respect to the rise of pulses, in spite of a band-limited pulse width by a $100 \mathrm{MHz}$ storage oscilloscope. The laser pulse followed the pumping $\mathrm{N}_{2}$ laser pulse, while the fluorescence one exhibited slower rise.

In order to verify that the laser operation was due to WGM, we also examined the mode structure of the emission spectra from a mixed-dye-doped polystyrene sphere. Figure 2 shows the typical emission spectra, which are obtained from a mixed-dye-doped sphere of the RH-101 (longer) and NA-F (shorter spectral regions) with the 50 $\mu \mathrm{m}$ diameter. For comparison, the typical emission spectra from an individual DD-WGM laser of RH-101 (red) and NA-F (blue regions), with the 100 and $65 \mu \mathrm{m}$ diameters, respectively, are shown in Fig. 3. The lasing spectra exhibit the rather simple periodic structure, which may be restricted by a resolution $(\sim 1 \mathrm{~nm})$ of the OMA system. The individual DD-WGM of RH-101 (in the upper) and NA-F (in the lower) oscillate in the red and green spectral regions, respectively, in Fig. 3, while the mixed-dye-doped sphere of RH-101/NA-F oscillates in both the green and orange spectral regions in Fig. 2. In the present characteristics from the mixed-dye-doped sphere of RH-101/NA-F, the emission spectra from RH-101 appear to be shorter wavelength shifted (red to orange) compared with those
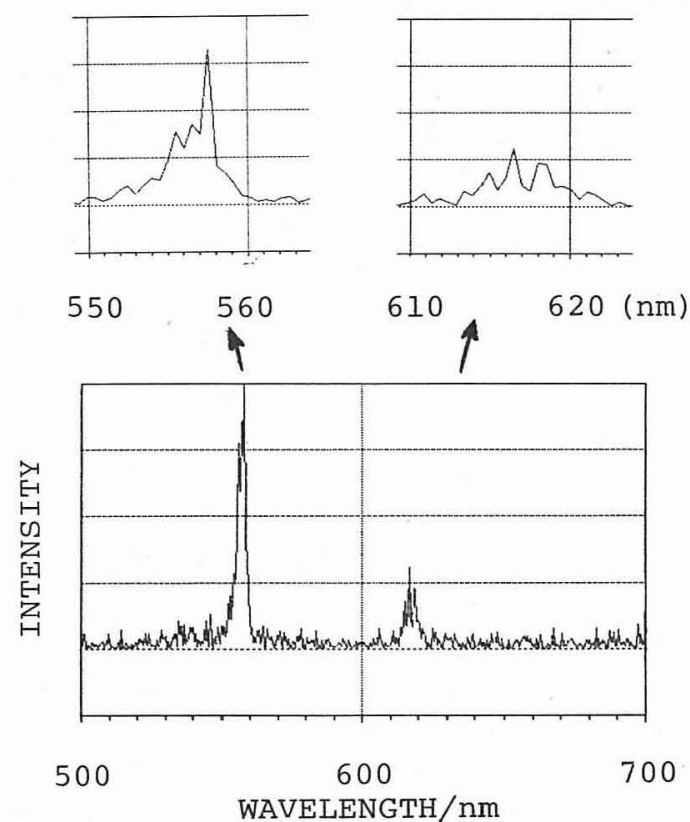

FIG. 2. Typical WGM spectra obtained from the sphere doped by the RH-101/NA-F mixed-dye ethanol solution with the $50 \mu \mathrm{m}$ diameter.
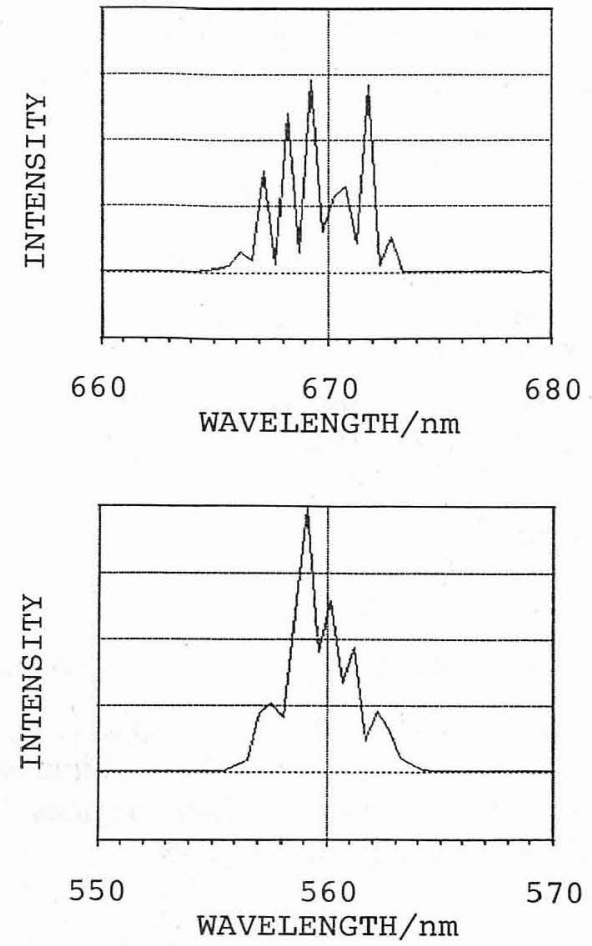

FIG. 3. Typical WGM spectra obtained from the spheres of the RH-101 (in the upper) and NA-F (in the lower) with the 100 and $65 \mu \mathrm{m}$ diameters, respectively.

from the individual one. However, these spectral shifts seem to be dependent with the mixture ratio of the both dyes similarly to be in usual liquid dye lasers.

The mode spacing of the observed spectra under the various diameters of the mixed-dye-doped WGM laser is found to be nearly proportional to the inverse diameter of the sphere. This indicates that the present laser operates according to the WGM in the spherical cavity.

In the smaller diameter of the focused excitation beam than that of the DD-WGM, no efficient laser operation was observed. The present data were obtained on a single shot; however, photobleaching of the DD-WGM by the excitation beam was observed on about 10 shots. In contrast with this fact, the photobleaching effect was not striking in the preliminary work with the Nd:YAG second-harmonic light-pulse $(532 \mathrm{~nm})$ excitation.

In conclusion, we have examined the emission from mixed-dye-doped solid microspheres pumped by a TEA UV $\mathrm{N}_{2}$ laser. The multiple oscillation of the solid spherical WGM laser output in both the green and orange spectral regions, from the mixed-dye-doped solid microspheres. The technique of the mixed-dye-doped spherical WGM dye laser reported here may be applied for obtaining various spectra, and/or an adequate selection of dye/solvent system having gain curves with close spectra may much expand oscillating spectral regions compared with those from an individual dye-doped microsphere. An investigation will be undertaken with the energy transfer effects between dyes in mixed-dye-doped solid particles.

The authors would like to express their thanks to Professor H. Inaba of Tohoku Technical University for his 
helpful suggestions. This work was partly supported by the 1991 Grant-in-Aid of the Special Project for Educational Research in Iwate University.

${ }^{1}$ C. G. Garrett, W. Kaiser, and W. L. Bond, Phys. Rev. 124, 1807 (1961).
${ }^{2}$ H.-M. Tzeng, K. F. Wall, M. B. Long, and R. K. Chang, Opt. Lett. 9, 499 (1984).

${ }^{3}$ H.-B. Lin, A. L. Huston, B. L. Justus, and A. J. Campillo, Opt. Lett. 11, 614 (1986)

${ }^{4}$ R. E. Benner, P. W. Barber, J. F. Owen, and R. K. Chang, Phys. Rev. Lett. 44, 475 (1980).

${ }^{5}$ M. K. Gonokami, K. Takeda, H. Yasuda, and K. Ema, Jpn. J. Appl. Phys. 31, L99 (1992). 